\title{
Oral Cystic Lymphangioma with Severe Respiratory Distress in a Neonate
}

\author{
${ }^{1}$ Kamlesh K Tiwari, ${ }^{2}$ Chetan Sharma, ${ }^{3}$ Vinita Chaturvedi, ${ }^{4}$ Ramesh Tangar, \\ ${ }^{5}$ Atul Ameta* \\ ${ }^{1,2,3,4}$ From the Department of Paediatric Surgery and Department of Gen.Surgery*, S.M.S. Medical College \& \\ associated SPMCHI, Jaipur-302004, India.
}

\begin{abstract}
Lymphangiomas are benign hamartomatous malformation of lymphatic vessels which contain clear protein rich fluid with few lymphcells. These malformations can occur at any age and involve any part of the body but have marked predilection to occur with head \& neck region (50 to 70\%). Occurrence of oral lymphangioma is rare and the tongue involvement is even rarer [1]. The Prognosis of lymphangiomas is good in most patient but respiratory obstruction is a feared complication of these lesion at head, neck and oral region as seen in our case, which may be fatal if not managed promptly.
\end{abstract}

Key words: Complication, Oral cystic lymphangioma, Respiratory distress.

\section{Introduction}

Lymphangiomas are a developmental malformation arising from sequestration of lymphatic tissue that do not communicate with the rest of the lymphatic system. They are benign lesion which neither become malignant nor have a familial tendency. About $50 \%$ of these lesions are noticed at birth and around $90 \%$ is developed before 2 years of age[2]. Lymphangiomas have been classified into three types based on microscopic characteristic of the lymphatic vessels diameter. These are : Capillary or microcystic, Cavernous or macrocystic and cystic or hygroma. A forth sub-type hemangiolymphangiomas also recognized with a vascular component[3]. These lesions are congenital or acquired which may exists either in isolation or associated with Down's syndrome, Turner's Syndrome Noonan's syndrome, Cardiac anomalies, Fetal hydrops, Fetal alcohol syndrome and Familial pterygium colli[4]. Oral lymphangiomas rare in occurrence and they are frequently located at the anterior $2 / 3$ of the tongue. Most lesions are benign and they have no chance of becoming malignant, are generally treated for cosmetic reasons only. Rarely, impingement upon critical organs leads to complications like respiratory distress or death due to the infiltration or compression of a normal airway by large cysts. Complete surgical excision is the treatment of choice but others are aspiration of cyst, laser and radiofrequency ablation, cryotherapy and sclerotherapy. We here in report a case of complicated oral cystic lymphangioma in a newborn with severe respiratory distress which was managed successfully.

\section{Case Report}

A newborn male child reported to the department of pediatric surgery at J.K. Lon hospital (SPMCHI) Jaipur with a large swelling protruding from oral cavity associated with severe respiratory distress since birth(Fig. 1 \&2). Family history was not significant.

On examination the patient was cyanosed with labored respiration. There was a large horrible looking swelling protruding from the oral cavity involving ventral part of the tongue. Swelling was completely occupied in the oral cavity and was cystic in nature with positive transillumination. His vitals were within normal limit except low $\mathrm{PaO}_{2}$.

\section{Management}

New born was admitted in ICU and started I/V fluid and Oxygen inhalation by hood. With a wide bore needle lesion was aspirated with aseptic precaution and about 50cc clear fluid was aspirated. After the aspiration swelling was collapsed (Fig. 3) which leads to abolish the pressure effect of the lesion on the upper air way. $\mathrm{PaO}_{2}$ and respiratory distress was improved. Routine blood investigations were within normal limit. On next day new born was taken up for definitive surgery. Lesion was arising from under surface of anterior $2 / 3$ of the tongue. Complete excision of this cystic lymphangioma was done (Fig. 4 \& 5). Post operatively the baby recovered well (Fig. 6). and was discharged uneventfully on $7^{\text {th }}$ day. The patient was followed up for 2 years, there was no recurrence (Fig. 7). 


\section{Discussion}

According to the size of the cysts lymphangiomas may be classified into Microcystic (volume of Cyst is $\left\langle 2 \mathrm{~cm}^{3}\right.$ ), Macrocystic (cyst volume $>2 \mathrm{~cm}^{3}$ ) and Mixed lymphangiomas which contain both microcystic and macrocystic components. It affects both sex equally and incidence is 1-3 cases/1000 live birth. It was reported that approximately 52 to $70 \%$ of all cases occur in head and neck region but the oral cavity rarely represents an occurrence site. Different treatment modalities for lymphangiomas are, aspiration, surgical excision, radiation therapy, electrocautery, cryotherapy, sclerotherapy, steroid therapy, embolisation, and ligation, laser surgery with ND-YAG, $\mathrm{CO}_{2}$ and radio frequency tissue ablation technique. Complete surgical excision is treatment of choice for lymphangiomas to prevent recurrence, because of finger like projections at periphery [5]. In the present case oral cystic lymphangioma compress the upper air way causing respiratory difficulty for new born. So in such emergency condition needle aspiration was done to decrease the pressure effect of the lesion on upper air way. Later on definitive treatment, complete surgical excision was performed to prevent recurrence. So early diagnosis \& treatment will help us to prevent complication of this large cystic lymphangioma. In sclerotherapy, Intralesional injection of sclerosing agents will result in inflammation, sclerosis \& cicatricial contraction of the lesion. Presently OK - 432 (Picibanil) is the preferred more effective and safe sclerosant for nonoperative and with macrocystic components of a lymphatic malformation[6].

\section{Conclusion}

Lymphangiomas are benign lesion and most of these having good prognosis but respiratory obstruction may be dreaded complication at head, neck and oral region which should manage promptly. Because of the non encapsulated and infiltrating nature complete surgical excision is treatment of choice otherwise recurrence may develop.

\section{References}

[1]. Mehar R, Garg A, Raj A, Singh I. Lympangioma of Tongue. the internet journal of otorhinolaryngology. 2005; 3(2).

[2]. S. Sunil, Devi Gopa Kumar, and B.S. Shrinivasan, Oral Lymphangioma. The contemporary clinical dentistry 2012 Jan-Mar; 3(1): 116-118.

[3]. Giguere CM, Bauman NM, Smith RJ (December 2012). "New Tretment Options for Lymphangioma in infant and children." The Annals of Otology, Rhinology and Laryngology 111 (12pt 1): 1066-75.

[4]. V Naveen Shanker, Ashwini. N Shankar, Praveena V. Shanlar J. Lymphvasculogenesis and Lymphangioma an update. Cancer Sci Ther. 2011; 3(6): 149-153.

[5]. Emery PJ, Bailey CM, Evans JN. Cystic Hygroma of Head and Neck : A review of 37 cases. J Laryngol Otol 1984; 98: 613-9.

[6]. Ahadi MMS. Lymphangioma of the tongue. Medical Journal of the Iranian Hospital. 1999'2 (1): 34-36.

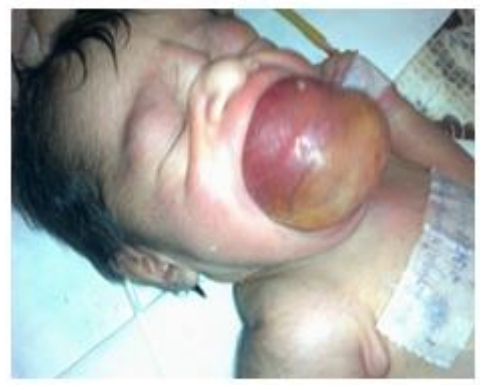

Fig. 1

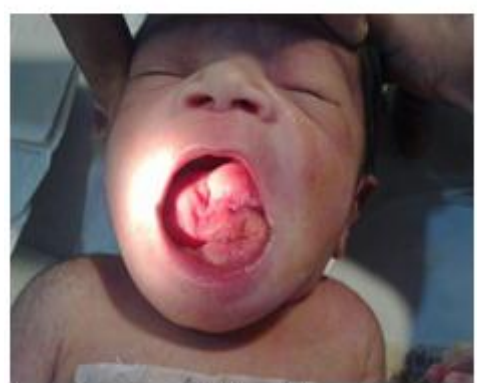

Fig. 3

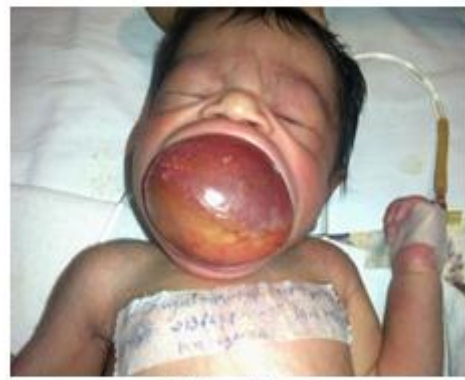

Fig. 2

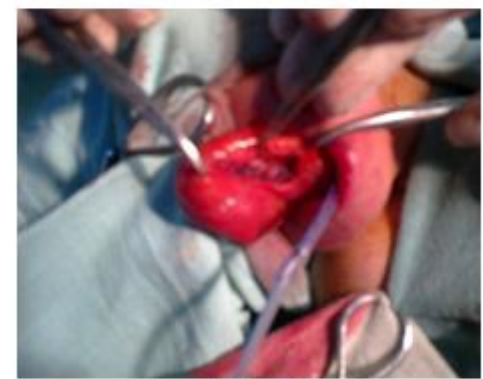

Fig. 4 


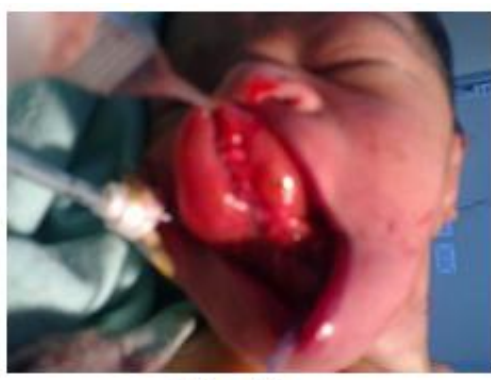

Fig. 5

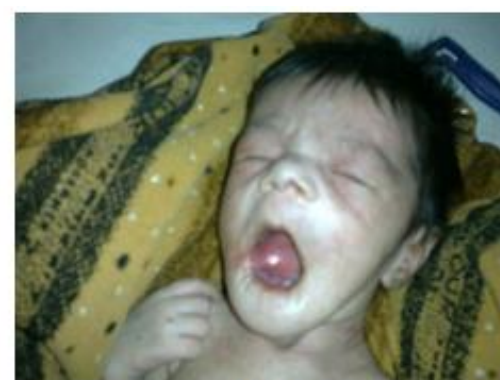

Fig. 6

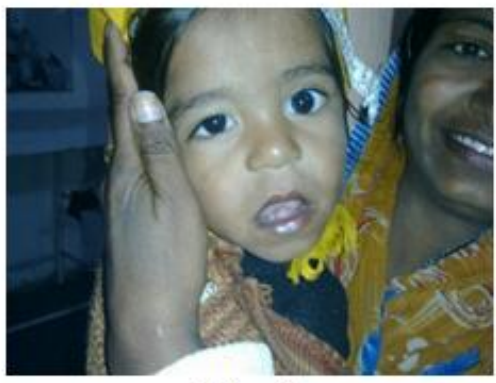

Fig. 7 